\title{
Choline Fenofibrate
}

National Cancer Institute

\section{Source}

National Cancer Institute. Choline Fenofibrate. NCI Thesaurus. Code C75253.

A choline formulation of fenofibrate, a synthetic phenoxy-isobutyric acid derivate and prodrug with antihyperlipidemic activity. 